\title{
Limitation on the accelerating gradient of a wakefield excited by an ultrarelativistic electron beam in rubidium plasma
}

\author{
N. Vafaei-Najafabadi, ${ }^{1 *}$ K. A. Marsh, ${ }^{1}$ C. E. Clayton, ${ }^{1}$ W. An, ${ }^{1}$ W. B. Mori, ${ }^{1}$ C. Joshi, ${ }^{1}$ W. Lu, ${ }^{2,1}$ \\ E. Adli, ${ }^{3,4}$ S. Corde,${ }^{3,5}$ C. I. Clarke, ${ }^{3}$ M. Litos, ${ }^{3}$ S. Z. Green, ${ }^{3}$ S. Gessner, ${ }^{3}$ J. Frederico, ${ }^{3}$ \\ A. S. Fisher, ${ }^{3}$ Z. Wu, ${ }^{3}$ D. Walz, ${ }^{3}$ and M. J. Hogan ${ }^{3}$ \\ ${ }^{1}$ University of California Los Angeles, Los Angeles, California 90095, USA \\ ${ }^{2}$ Tsinghua University, Beijing 100084, China \\ ${ }^{3}$ SLAC National Accelerator Laboratory, Menlo Park, California 94025, USA \\ ${ }^{4}$ Department of Physics, University of Oslo, 0316 Oslo, Norway \\ ${ }^{5}$ LOA, ENSTA ParisTech, CNRS, Ecole Polytechnique, Universite Paris-Saclay, Palaiseau 91762, France
}

(Received 15 July 2016; published 25 October 2016)

\begin{abstract}
We have investigated the viability of using plasmas formed by ionization of high $\mathrm{Z}$, low ionization potential element rubidium $(\mathrm{Rb})$ for beam-driven plasma wakefield acceleration. The $\mathrm{Rb}$ vapor column confined by argon (Ar) buffer gas was used to reduce the expected limitation on the beam propagation length due to head erosion that was observed previously when a lower $\mathrm{Z}$ but higher ionization potential lithium vapor was used. However, injection of electrons into the wakefield due to ionization of Ar buffer gas and nonuniform ionization of $\mathrm{Rb}^{1+}$ to $\mathrm{Rb}^{2+}$ was a possible concern. In this paper we describe experimental results and the supporting simulations which indicate that such ionization of $\mathrm{Ar}$ and $\mathrm{Rb}^{1+}$ in the presence of combined fields of the beam and the wakefield inside the wake does indeed occur. Some of this charge accumulates in the accelerating region of the wake leading to the reduction of the electric field - an effect known as beam loading. The beam-loading effect is quantified by determining the average transformer ratio $\langle R\rangle$ which is the maximum energy gained divided by the maximum energy lost by the electrons in the bunch used to produce the wake. $\langle R\rangle$ is shown to depend on the propagation length and the quantity of the accumulated charge, indicating that the distributed injection of secondary Rb electrons is the main cause of beam loading in this experiment. The average transformer ratio is reduced from 1.5 to less than 1 as the excess charge from secondary ionization increased from 100 to $700 \mathrm{pC}$. The simulations show that while the decelerating field remains constant, the accelerating field is reduced from its unloaded value of 82 to $46 \mathrm{GeV} / \mathrm{m}$ due to this distributed injection of dark current into the wake.
\end{abstract}

DOI: 10.1103/PhysRevAccelBeams.19.101303

\section{INTRODUCTION}

The first phase of research on plasma wakefield accelerators (PWFAs) at the Final Focus Test Beam (FFTB) of the SLAC National Accelerator Laboratory demonstrated the ability of a PWFA to achieve ultrahigh acceleration gradients of over $50 \mathrm{GV} / \mathrm{m}[1]$ in a meter scale plasma. The experiments were carried out in the so-called blowout regime $[2,3]$, where the electron beam density, $n_{b}$, was larger than the plasma density, $n_{p}$. In this regime, the drivebeam electrons expel plasma electrons, creating an ion cavity. A trailing electron beam placed inside this cavity will experience a linear focusing force, which preserves its transverse phase space and emittance, while (at the right phase) experiencing an accelerating longitudinal field. The maximum accelerating field is given by [2]

\footnotetext{
*Now at Stony Brook University.

Published by the American Physical Society under the terms of the Creative Commons Attribution 3.0 License. Further distribution of this work must maintain attribution to the author $(s)$ and the published article's title, journal citation, and DOI.
}

$$
e E_{\text {peak }} /\left(m c \omega_{p}\right)=-\frac{1}{2} k_{p} R_{b}
$$

where $R_{b}$ is the maximum blowout radius, $m$ is the electron mass, $e$ is the electron charge, $c$ is the speed of light, and $k_{p}=\omega_{p} / c$ is the inverse skin depth of the plasma. In a bi-Gaussian beam with $k_{p} \sigma_{z} \approx 1, k_{p} \sigma_{r} \ll 1$,

$$
k_{p} R_{b} \approx 2 \sqrt{\Lambda}
$$

where $\Lambda$ is the normalized charge per unit length given by $\Lambda=\frac{n_{b}}{n_{p}}\left(k_{p} \sigma_{r}\right)^{2}$. For a bi-Gaussian beam, $n_{b}=\frac{N}{(2 \pi)^{3 / 2} \sigma_{z} \sigma_{r}^{2}}$, where $N$ is the number of electrons in the bi-Gaussian bunch and $\sigma_{r}$ and $\sigma_{z}$ are the rms bunch width and bunch length, respectively [4]. Thus for the parameters used in [1], i.e. a plasma density of $n_{p}=2.7 \times 10^{17} \mathrm{~cm}^{-3}$ and a drive beam with charge of $N=1.8 \times 10^{10}$ electrons and $\sigma_{z}=15$ microns, the expected gradient given by Eq. (1) is $82 \mathrm{GV} / \mathrm{m}$. In the experiment the observed maximum gradient can be smaller than the above value if the electrons bunch is not long enough to probe the highest value of the 
accelerating field as was the case in Ref. [1]. Nevertheless, electrons in the tail of the Gaussian drive beam were accelerated from an initial energy of $42 \mathrm{GeV}$ (produced by the $3 \mathrm{~km}$ SLAC linac) to $85 \mathrm{GeV}$, i.e. some electrons doubled their energy in just over $80 \mathrm{~cm}$, representing an average accelerating gradient of $54 \mathrm{GeV} / \mathrm{m}$.

The interaction length in the above-mentioned selfionized PWFA experiment was limited by "head erosion." In self-ionization, the plasma is produced by the transverse electric field of the front (head) of the highly relativistic beam that also drives the plasma wake. Since the plasma electrons are expelled over a distance of $\frac{2}{k_{p}} \sqrt{\frac{n_{p}}{n_{b}}}$ [5], the blowout region, which is the region where electrons experience focusing force, forms behind the ionizing head of the beam. Therefore, the ionizing head of the beam does not experience this focusing force and expands due to the beam's inherent emittance. This expansion in turn reduces the transverse electric field at the head of the beam and as a consequence, the ionization front continuously moves back in the frame of the beam during the interaction. This effect is called beam head erosion [6], and in the extreme case, it can result in an interaction length that is smaller than the beam's $\beta$ function in vacuum.

Head erosion is undesirable because it can effectively reduce the length over which the wake can be formed to a distance smaller than the pump depletion length-when the energy of the drive bunch particles is almost depleted and the bunch is no longer able to excite a wake. The head erosion speed of a matched beam (matching is defined later in the paper), $V$, depends on the normalized beam emittance $\epsilon_{n}$, its current $I$, the beam energy $\gamma_{b}$, and the ionization potential (IP) of the medium being ionized [5-7] according to

$$
V[\mu \mathrm{m} / m] \propto \mathrm{IP}^{1.73}[\mathrm{eV}] \epsilon_{N}[\mathrm{~mm}-\mathrm{mrad}] \gamma_{b}^{-1} I^{-3 / 2}[\mathrm{kA}] .
$$

For a given drive bunch, one can reduce the head erosion speed and thereby achieve a longer interaction length by using a medium with lower ionization potential. Therefore, to potentially achieve larger interaction length than in Ref. [1], one could replace the lithium plasma source with one of the handful of elements that have a lower ionization potential. For example, the ionization potential (IP) of the first electron of $\mathrm{Rb}$ is $4.4 \mathrm{eV}$ (compared to $5.4 \mathrm{eV}$ for $\mathrm{Li}$ ), so the SLAC electron beam is expected to propagate $40 \%$ further (and therefore give a correspondingly higher energy gain) in the beam-ionized $\mathrm{Rb}$ plasma compared to the previously used Li plasma according to Eq. (3).

This choice of $\mathrm{Rb}$ is also expected to reduce the problem of emittance growth of the accelerating beam due to ion motion $[8,9]$ in future PWFAs. In the present experiments, the plasma ions are assumed to remain stationary on the scale of the bunch length. However, in future experiments, it is expected that $n_{b} / n_{p}>m_{i} / m_{e}$, where $m_{i}$ and $m_{e}$ are the ion and electron mass, respectively. In such a case, the
Coulomb force of the drive or the accelerating trailing electron bunch will cause the plasma ions to move toward the axis, degrading the linear focusing force of the ion cavity, and leading to the emittance dilution of the electrons. Since $\mathrm{Rb}$ atoms are 10 times heavier than the $\mathrm{Li}$ atoms, the use of $\mathrm{Rb}$ is expected to help mitigate the ion motion problem for high beam density experiments. It should be noted that even with the higher $\mathrm{Z}$ of the $\mathrm{Rb}$ ions, emittance growth due to small angle (electron-ion) scattering is not a serious issue for electron beam energies greater than $10 \mathrm{GeV}$ [10].

For the two reasons given above, we tested a Rb plasma source for PWFA experiments at FACET. However, we expected that the maximum acceleration gradient of the wakes in $\mathrm{Rb}$ plasma may be smaller than the $\mathrm{Li}$ source. The origin of this effect is the smaller ionization potential of the $\mathrm{Rb}^{1+}$ (IP $27.3 \mathrm{eV}$ ) compared to $\mathrm{Li}^{1+}$ (IP 75.6 eV). For a beam with $\sigma_{z}=40 \mu \mathrm{m}$, the threshold field for the ionization of neutral $\mathrm{Rb}$ and $\mathrm{Li}$ are 3.0, $4.7 \mathrm{GV} / \mathrm{m}$, and for $\mathrm{Rb}^{1+}$, and $\mathrm{Li}^{1+}$ are 52 and $290 \mathrm{GV} / \mathrm{m}$, respectively. Here, ionization threshold is defined as when the beam ionizes $10 \%$ of neutral atoms. The method for calculation of these values is explained later, but the important point is that the onset of the ionization of $\mathrm{Rb}$ ion can occur at a much lower electric field than for the $\mathrm{Li}$ ion.

Furthermore, the electrons resulting from the ionization of the $\mathrm{Rb}^{1+}$ ion ( $\mathrm{Rb}$ II electrons) are born inside the wake and can be accelerated by it. The ionization of the $\mathrm{Rb}^{1+}$ ion within the wake occurs as a result of the combined effect of the wakefields and the beam field even if the beam field alone is below the threshold value for field-induced ionization. This injection of charge may be quite localized if the drive bunch is initially not matched to the plasma. As it will be shown later, if the drive bunch radius is larger than the matched radius, the bunch undergoes periodic envelope oscillations. Thus the ionization threshold of $\mathrm{Rb}^{1+}$ may be exceeded in the tightly pinched regions of each oscillation of the electron beam envelope [11] in the plasma, leading to the distributed injection of what amounts to "dark current."

While the distributed injection of this charge does not affect the decelerating field because of causality, it does reduce the accelerating field because of the beam loading effect [12] and reduce the average transformer ratio $\langle R\rangle$ [2]. In the experiments described here, $\langle R\rangle$ is reduced from its weakly loaded value of about 1.5 to 0.75 . In the simulation, the reduction of the $\langle R\rangle$ is shown to be associated with the reduction of the maximum accelerating gradient from an unloaded value of $82 \mathrm{GeV} / \mathrm{m}$ to about $46 \mathrm{GeV} / \mathrm{m}$. This problem is not unique to $\mathrm{Rb}$. Indeed, other alkali elements with lower ionization potential considered to replace lithium in plasma wakefield acceleration face the same problem.

\section{BEAM LOADING AND TRANSFORMER RATIO}

An important figure of merit for a beam-driven PWFA is the transformer ratio $R$, which is the ratio of the peak accelerating field to the peak decelerating field, i.e. 


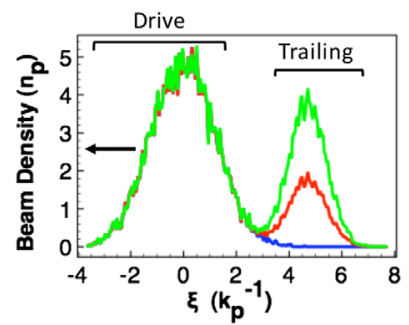

(a)

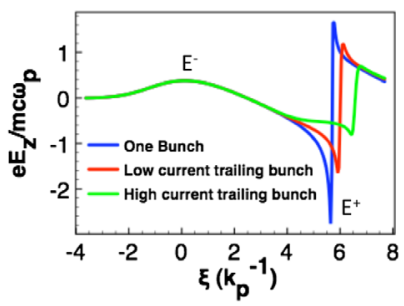

(b)
FIG. 1. Figure originally appeared in [16]. Beam loading in a drive/trailing electron-driven plasma wakefield accelerator. (a) The longitudinal density profile of drive and trailing electron beams in three different beam loading scenarios. The drive beam density is the same $\left(n_{b} \sim 5 n_{p}\right)$ in all three cases. The three cases of trailing beam are: no trailing beam, i.e. unloaded wake (blue), trailing beam with peak density $40 \%$ that of the drive bunch (red), and trailing beam with peak density $80 \%$ that of the drive bunch (green). (b) The on-axis longitudinal electric field for each of the three cases in (a). One can see progressively stronger beam loading that leads to flattening of the wake and reduction in the transformer ratio as described in the text.

$R=E^{+} / E^{-}$. In a nonevolving wake, this ratio also indicates the energy gained by a trailing beam or a "beam load" in the accelerator to the energy lost by the drive beam. Since the total energy gained by the beam load cannot exceed the energy lost by the drive beam, the number of particles in the beam load must be less than $1 / R$ of the number of particles in the drive beam. In other words, in a plasma wakefield accelerator, there is an inverse relation between the charge and the energy of the beam load, in an analogous way to the relationship between current and voltage on a load in a transformer-hence the term transformer ratio. It is well known that in the linear regime of the PWFA, $R$ is less than 2 for symmetric beams and unloaded wakes $[13,14]$, which means that $20 \mathrm{GeV}$ electrons in the drive beam can at most add $40 \mathrm{GeV}$ energy to electrons that are being accelerated. In the nonlinear regime, $R>2$ may be possible to obtain by the careful shaping of drive and trailing beams [15].

We first give a physical picture of beam loading and its effect on $R$ since we shall use the average transformer ratio $\langle R\rangle$ to quantify the extent of beam loading in this work. As shown in Fig. 1, the accelerating field of the wake can be locally flattened and reduced by the particles comprising the beam load. The beam load may be provided by a second trailing bunch or it may be "dark current" injected from the plasma itself or some combination of the two.

Wake flattening due to beam loading is a necessary and indeed a very desirable effect when it is caused by a second (trailing) bunch that is being accelerated. This is because flattening of the wake helps to reduce the energy spread. It is also necessary for an efficient energy extraction from the wake by the trailing beam as the energy previously stored in the electric field of wake is absorbed by the beam load. However, severe intentional or unintentional (dark current) beam loading will damp $E^{+}$and drastically reduce $R$. For plasma wakes in the blowout regime, the amount of charge that is required to reduce the accelerating field of the wake from $E_{\text {peak }}$ [as given by Eq. (1)] to a flat value of $E_{s}$ can be estimated using [12]

$$
Q[\mathrm{nC}]=\frac{0.047 m c \omega_{p}}{e E_{s}} \sqrt{\frac{10^{16}}{n_{p}}}\left(k_{p} R_{b}\right)^{4} .
$$

Using the parameters that will be relevant to the experiment, where plasma density $n_{p}=2.7 \times 10^{17} \mathrm{~cm}^{-3}$, the initial beam has $N=1.8 \times 10^{10}$ particles with $\mathrm{rms}$ bunch length of $\sigma_{z}=40 \mu \mathrm{m}$, Eq. (1) can be used to obtain the values of $k_{p} R_{b}=2.0$ and $E_{\text {peak }}=50.2 \mathrm{GV} / \mathrm{m}$. Therefore, reducing the peak accelerating field to $E_{\text {peak }} / 2$ requires $Q>300 \mathrm{pC}$ and reducing it to $E_{\text {peak }} / 4$ requires $Q>600 \mathrm{pC}$ according to Eq. (4). Note that this value is calculated for a charge with specific shape that produces a uniformly flat accelerating field for $\xi>\xi_{i}$, where $E\left(\xi_{i}\right)=E_{s}$ [similar to the case of the green curve in Fig. 1(b)]. It is therefore possible for a wake to trap a much larger amount of charge if beam loading is nonuniform. Nevertheless, the values of Q calculated here give an indication of the amount of the charge that would cause heavy beam loading.

\section{IONIZATION INJECTION INTO THE PLASMA WAKEFIELD}

An important problem in the field of plasma wakefield acceleration is to place an electron bunch-or the beam load-at an appropriate position in the accelerating phase of the plasma wake. The issue is that the wakefield moves through the plasma at a phase velocity equal to the velocity of the electron beam driver. For the $20.35 \mathrm{GeV}$ electron beam at FACET, that represents a phase velocity with $\gamma_{b} \approx 40,000$. Therefore, the beam load needs to be injected into the plasma wakefield with high enough $\gamma_{b}$ such that it can remain in the accelerating structure for a long distance. The process of electrons being positioned inside the wakefield with sufficient energy that will enable acceleration over a long distance is referred to as injection.

One method of injection into the wakefield is external injection. In this method, the second electron beam is separately prepared and accelerated to a sufficient energy such that it can move synchronously with the wake. This method was used at FACET to create a secondary bunch that extracted energy from a plasma wakefield at a gradient of $5 \mathrm{GeV} / \mathrm{m}$ and at efficiency of up to $30 \%$ [17].

The other method of injection is known as ionization injection. This method uses large separation between the ionization potentials of two elements to produce electrons within a fully formed wake $[18,19]$. The electrons from the element with the lower ionization potential are used to form the wake, while the element with the higher ionization potential is ionized within the wake, where the high 
accelerating field can impart enough energy to these electrons such that they travel near the phase velocity of the wake. The physics of ionization injection for such electrons was described by $[18,19]$. The constant of motion for an electron interacting with the wakefield can be shown to be $\bar{H}=\gamma_{b} m c^{2}-v_{\phi} P_{z}-e \Psi$, where $v_{\phi}$ is the phase velocity of the wake, $\Psi \equiv \Phi-\frac{v_{\phi}}{c} A_{z}$ is defined as the wake potential, where $\Phi$ is the electrostatic potential and $A_{z}$ is the $\mathrm{z}$ component of the vector potential. For an electron born at rest with some initial $\Psi$ to become trapped, i.e. $v_{z}=v_{\phi}$,

$$
\begin{gathered}
\bar{H}_{i}=\bar{H}_{f}, \\
1-\frac{e \Psi_{i}}{m c^{2}}=\gamma_{b}-\frac{v_{\phi} P_{z}}{m c^{2}}-\frac{e \Psi_{f}}{m c^{2}}, \\
\Delta \bar{\Psi}+1=\frac{\gamma_{b}}{\gamma_{\phi}}=\frac{\sqrt{1+\left(P_{\perp} / m c\right)^{2}}}{\gamma_{\phi}} .
\end{gathered}
$$

Here, $\Delta \bar{\Psi}=\frac{e}{m c^{2}}\left(\Psi_{f}-\Psi_{i}\right)$ and $\gamma_{\phi}=\left[1-\left(v_{\phi} / c\right)^{2}\right]^{-1 / 2}$. In the experiments, $\gamma_{\phi} \sim \gamma_{b} \sim 40,000$, meaning that transverse momentum remains much smaller than the longitudinal momentum, and the trapping condition simplifies to $\Delta \bar{\Psi} \leq-1$.

As it will be shown in the simulation section, this condition is only satisfied for electrons that are ionized within the wake cavity (also discussed in Ref. [20]). In other words, only for electrons that are born inside the wake is there a chance to get trapped by such a high-phase-velocity wake. In the present experiment, this ionization occurs via field ionization, because as will be shown later, the combined field of the wake and the evolving beam is strong enough to ionize Ar, $\mathrm{Ar}^{1+}$ and $\mathrm{Rb}^{1+}$ electrons, which will lead to their trapping since they can satisfy $\Delta \bar{\Psi} \leq-1$.

\section{EXPERIMENTAL SETUP}

This experiment was performed at the FACET facility [21] at the SLAC National Accelerator Laboratory. The schematic of the experimental setup is shown in Fig. 2. The $3 \mathrm{nC}, 20.35 \mathrm{GeV}$ electron beam was focused on the rising density edge of a column of $\mathrm{Rb}$ vapor, which was generated in a heat-pipe oven [22]. The density profile of the $\mathrm{Rb}$ in the heat pipe oven is shown as an inset in Fig. 2. Also shown is the density of the Ar buffer gas, which confines the Rb to the hot region of the oven by cooling the $\mathrm{Rb}$ atoms via collisions. Accordingly, the density of Ar rises as the $\mathrm{Rb}$ density falls and vice versa.

The important beam and plasma parameters of the experiment are shown in Table I. The initial electron beam density is less than the plasma density, which means that the blowout regime is not immediately established. Nevertheless, the beam electrons still repel some of the plasma electrons, creating a region of positive charge density. The focusing force of this partial blowout region $\begin{array}{rlr}\delta \mathrm{p} / \mathrm{p}(\%) \\ -2 & 0 & 2\end{array}$

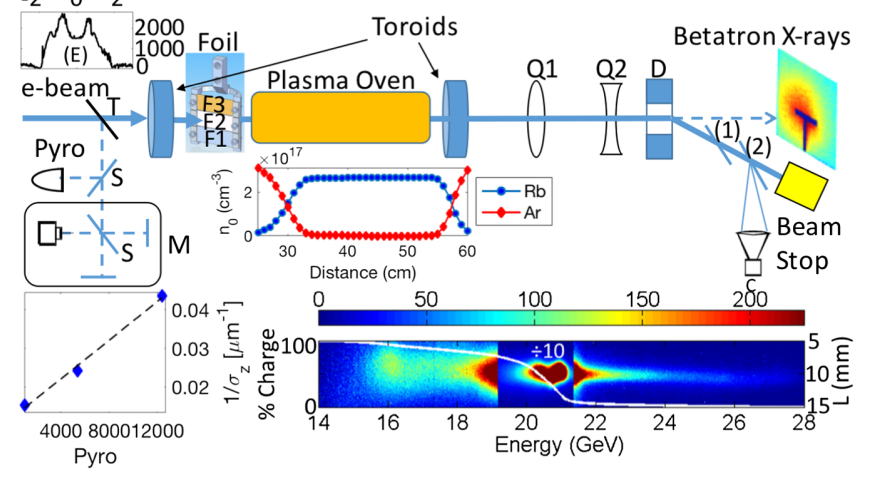

FIG. 2. Experimental setup. The electron beam enters from the left. The energy spectrum of the beam before plasma is shown as the inset (E) on the top left. The transition radiation generated as the beam traverses the $1 \mu \mathrm{m}$ Ti foil (T) is observed simultaneously by a pyroelectric detector (Pyro) and a $\mathrm{THz}$ Michelson interferometer $(\mathrm{M})$. Correlation between the pyro and the inverse of the bunch length deduced from the Michelson interferometer is shown beneath the setup. Foils with progressively higher scattering strength (F1-F3) can be inserted in the path of the beam before it enters the plasma oven, leading to progressively increased beam emittance (see Table II for details). The density profiles of neutral $\mathrm{Rb}$ and $\mathrm{Ar}$ are plotted underneath the schematic of the oven in blue circles and red diamonds, respectively. Absolutely calibrated toroids upstream and downstream of the plasma record the amount of charge entering and exiting the plasma. The quadrupole magnets Q1 and Q2 along with the dipole magnet $\mathrm{D}$ form the imaging energy spectrometer. The Cherenkov radiation emitted by the dispersed electrons between silicon wafers (1) and (2) is detected by camera $\mathrm{C}$ to give the electron spectrum after the plasma. An example of the energy spectrum charge is shown below the camera $\mathrm{C}$, with the white curve showing the integrated charge. The charge near $20.4 \mathrm{GeV}$ observed not to lose any energy has been attenuated by a factor of 10 . The Lanex screen, shielded by a $1 \mathrm{~mm}$ copper foil, detects the betatron $\mathrm{x}$ rays.

focuses the electron beam, increasing the drive beam's density, which in turn repels more plasma electrons. This feedback between the focusing force of the partially blownout ion column and the stronger repelling force of the focused drive electron beam continues until the blowout regime is established. In the simulations discussed later, the blowout regime is established in less than $3 \mathrm{~cm}$.

The normalized emittance of the beam in the experiment is manipulated by inserting foils (F1-F3) in the electron beam path $65.2 \mathrm{~cm}$ upstream of the plasma source and the waist. Because the head-erosion rate is a function of emittance [see Eq. (3)], these foils are used to change the head-erosion rate, and consequently the distance over which the wake is excited by the beam-or the interaction length. The scattering of the electron beam as it goes through a foil can be modeled using the rms multiple scattering angle $(\Delta \theta)$, which can be calculated using the standard formula [23]. Using this model, and the values of 
TABLE I. Plasma and beam parameters in the experiment. $n_{b}$ and $R_{b}$ are calculated with $\sigma_{z}=40 \mu \mathrm{m}$. $L_{\mathrm{FWHM}}$ is the full width at half maximum of the rubidium density profile (shown as an inset in Fig. 2).

\begin{tabular}{lc}
\hline \hline Parameter & Value \\
\hline Number of electrons in drive bunch & $1.8 \times 10^{10}$ \\
$\sigma_{z}(\mu \mathrm{m})$ & $39-60$ \\
$\sigma_{r}(\mu \mathrm{m})$ & 35 \\
$n_{b}\left(\mathrm{~cm}^{-3}\right)$ & $2.3 \times 10^{16}$ \\
$n_{p}\left(\mathrm{~cm}^{-3}\right)$ & $2.7 \times 10^{17}$ \\
$R_{b}(\mu \mathrm{m})$ & 21 \\
$L_{\mathrm{FWHM}}(\mathrm{cm})$ & 28 \\
\hline \hline
\end{tabular}

the vacuum waist and emittance $\sigma_{r}^{*}=35 \mu \mathrm{m}$ and $\epsilon_{n} \sim 250 \mathrm{~mm}$-mrad, respectively, the values of $\epsilon$, and Twiss parameters $(\alpha, \beta$, and $\gamma)$ can be calculated using

$$
\begin{gathered}
\epsilon=\epsilon_{n} / \gamma_{b}, \\
\beta=\sigma_{r}^{2} / \epsilon, \\
\gamma=\frac{1+\alpha^{2}}{\beta} .
\end{gathered}
$$

At the waist, $\alpha^{*}=0, \beta^{*} \sim 0.19 \mathrm{~m}$, and $\gamma^{*} \sim 5.1 \mathrm{~m}^{-1}$, where the $*$ denotes the values at waist. The propagation of Twiss parameters in vacuum can be calculated using

$$
\left[\begin{array}{l}
\beta \\
\alpha \\
\gamma
\end{array}\right]=\left[\begin{array}{ccc}
1 & -2 L & L^{2} \\
0 & 1 & -L \\
0 & 0 & 1
\end{array}\right]\left[\begin{array}{l}
\beta^{*} \\
\alpha^{*} \\
\gamma^{*}
\end{array}\right]
$$

At the location of foil, where $L=-65.2 \mathrm{~cm}$, i.e. $65.2 \mathrm{~cm}$ upstream of the plasma entrance, the Twiss parameters are $\alpha_{v}=3.3, \beta_{v} \sim 2.4 \mathrm{~m}$, and $\gamma_{v} \sim 5.1 \mathrm{~m}^{-1}$. Here, the subscript " $v$ " denotes the values in vacuum (i.e. no foils), and $L=-0.652 \mathrm{~m}$. The change in the Twiss parameters due to a foil is given by equations [24-26]

$$
\begin{gathered}
\epsilon_{f}^{2}=\epsilon_{v}\left(\epsilon_{v}+\beta_{v} \Delta \theta^{2}\right), \\
\beta_{f}^{2}=\beta_{v}^{2} \epsilon_{v} /\left(\epsilon_{v}+\beta_{v} \Delta \theta^{2}\right),
\end{gathered}
$$

where the subscript " $f$ " denotes the values at $L=-0.652 \mathrm{~m}$ in the presence of the foil. Finally, using Eqs. (13), (11), and (9), the value of the $\sigma_{r}$ at the entrance of the plasma after propagating through a foil can be calculated. The properties of these foils, and their effect on the emittance and $\sigma_{r}$ at the entrance of plasma are shown in Table II. Note that these emittance numbers are similar to those calculated in [27], which used the simulation code ELEGANT to calculate the value of the normalized emittance and $\sigma_{r}$ due to the foils.

Data from the following diagnostics, which were recorded for every data point, will be used in this paper: the charge monitor (toroids), the electron energy spectrum before and after plasma, the x-ray yield (betatron screen), and the bunch length $\left(\sigma_{z}\right)$ from the pyro detector, all of which are shown in Fig. 2.

The bunch length $\left(\sigma_{z}\right)$ was measured on every shot using a pyroelectric detector (pyro), which detected the coherent transition radiation (CTR) that was generated as the electron beam traversed a $1 \mu \mathrm{m}$ thick titanium foil. The CTR signal varies as $N^{2} / \sigma_{z}$ [28], and since the number of electrons, $N$, is nearly constant during the experiment, the variation in pyro correlates with a variation in $1 / \sigma_{z}$. This linear relationship between the pyro and inverse bunch length was confirmed in the region of interest using a $\mathrm{THz}$ Michelson interferometer [29], which could monitor the CTR simultaneously with the pyroelectric detector as shown in an inset in Fig. 2.

The incoming and outgoing charge was measured upstream and downstream of the plasma using toroidal current monitors-toroids. By comparing the charge upstream and downstream of the plasma, we measured any "excess charge" generated during the experiment.

The energy spectrum of the electron beam after the plasma was measured using an imaging spectrometer. The spectrometer included a dipole magnet, which bent and dispersed the electrons according to their energy, and a pair of quadrupole magnets, which focused electrons at a specific energy. As the dispersed electrons propagate through the air, they generate Cherenkov emission. This broadband visible light emitted by the electrons between a pair of $\mathrm{Si}$ wafers $5 \mathrm{~cm}$ apart was imaged by a lens onto a CCD camera to record the dispersed spectrum of the electrons. These dispersed spectra are analyzed for the

\begin{tabular}{|c|c|c|c|c|c|}
\hline Name & Composition & Thickness $(\mu \mathrm{m})$ & $\Delta \theta(\mu \mathrm{rad})$ & $\epsilon_{n}(\mathrm{~mm}-\mathrm{mrad})$ & $\sigma_{r}(\mu \mathrm{m})$ \\
\hline No foil & & $\ldots$ & $\ldots$ & 250 & 35 \\
\hline Foil 1 & Nicusil $3(71.15 \mathrm{Ag} / 28.1 \mathrm{Cu} / 0.75 \mathrm{Ni})$ & 25.4 & 35 & 302 & 58 \\
\hline Foil 2 & $25 / 75 \mathrm{Au} / \mathrm{Cu}$ & 50.8 & 58 & 377 & 64 \\
\hline Foil 3 & Nocoro $80(81.1 \mathrm{Au} / 16 \mathrm{Cu} / 2 \mathrm{Ni})$ & 38.1 & 64 & 399 & 66 \\
\hline
\end{tabular}
maximum energy gain and the maximum energy loss. The maximum energy gain $\left(\int E_{\max }^{+} d l\right)$ and the maximum energy loss $\left(\int E_{\max }^{-} d l\right)$ for each shot are defined as the $2 \%$

TABLE II. Properties of scattering foils used in the experiment and their effect on normalized emittance and $\sigma_{r}$ at the entrance of plasma. See text for how the normalized emittance is calculated. 
and $98 \%$ contours of the integrated Cherenkov signal, and an example is shown as a white curve on the spectrum in Fig. 2.

The $\mathrm{x}$ rays generated during the betatron oscillations $[30,31]$ by the electrons in the ion cavity of the wake were detected using a Lanex film after the plasma. To measure the $\mathrm{x}$-ray signal generated during the betatron motion only, the region of Lanex screen that showed radiation in the absence of plasma was blocked: This region is shown as a solid blue T-shaped region on the betatron x-rays diagnostic in Fig. 2. A significant portion of this blocked radiation comes from the upstream and downstream quadrupoles and other magnets in the beam line. The remaining $\mathrm{x}$-ray signal is mostly due to betatron emission. This $\mathrm{x}$-ray signal is then normalized for the amount of the beam charge that actually resides inside the wake and therefore generates betatron radiation. This "participating charge" is found by subtracting the charge that has lost no energy from the total amount of charge on the dispersed electron spectrum. This process is explained later in the paper. The resulting $\mathrm{x}$-ray yield is used as a measure of $\mathrm{x}$ rays generated per electron in the experiment.

Finally, the spectrum of the electron beam before the plasma was recorded in each shot. This spectrum was measured in a region of the linac with significant spatial dispersion resulting in a correlation between energy and the transverse coordinate. The beam is then sent through a magnetic chicane, resulting in emission of synchrotron radiation by these electrons. The radiation is captured using a screen of YAG:Ce crystal, and because of energy chirp of the beam, the brightness of the $\mathrm{x}$ rays corresponds to the current versus energy of electrons. This spectrum may be used for the recovery of the longitudinal beam profile using the LITRACK simulation code [28]. In these experiments however, the beam spectrum was merely used to isolate data points with nearly identical spectra (and therefore longitudinal profile) in different data sets. Additionally, because of the relationship between the pyro signal and the bunch length, the similar YAG spectra were seen within a narrow range of the pyro signal.

\section{PHYSICS OF DISTRIBUTED INJECTION}

Comparing the toroids upstream and downstream of the plasma shows that a large amount of excess charge $(\Delta Q)$ is generated during the experiment. The physics of generating this excess charge can be explained by exploring the beam evolution through the plasma.

In the absence of the foils (F1-F3), the electron beam enters the Rb gas column with $\sigma_{r} \sim 35 \mu \mathrm{m}$ and $\sigma_{z} \sim 40 \mu \mathrm{m}$. The value of $\sigma_{z}$ is inferred from the pyro signal using the calibration curve shown in Fig. 2. The value of $\sigma_{r}$ is measured using the SLAC wire-scanner diagnostic [32] near the beam waist.

The peak electric field of an electron beam with bi-Gaussian profile can be expressed as [33]

$$
E_{r}^{\max }=10.4\left[\frac{\mathrm{GV}}{\mathrm{m}}\right] \frac{N}{10^{10}} \frac{10}{\sigma_{r}[\mu \mathrm{m}]} \frac{50}{\sigma_{z}[\mu \mathrm{m}]},
$$

where $N$ is the number of electrons in the bunch. For the electron beam used in these experiments, $N=1.8 \times 10^{10}$. The ionization rate of an element due to this electric field can be accurately modeled using the Ammosov-Delone-Krainov (ADK) formalism [33,34]

$$
\begin{aligned}
& W\left(s^{-1}\right)= 1.5 \times 10^{15} \frac{4^{n} \mathrm{IP}(\mathrm{eV})}{n \Gamma(2 n)}\left[20.5 \frac{\mathrm{IP}^{3 / 2}(\mathrm{eV})}{E(\mathrm{GV} / \mathrm{m})}\right]^{2 n-1} \\
& \times \exp \left[-6.83 \frac{\mathrm{IP}^{3 / 2}(\mathrm{eV})}{E(\mathrm{GV} / \mathrm{m})}\right] \\
& n_{e}=n_{0}\left[1-\exp \left(-\int W d t\right)\right] .
\end{aligned}
$$

Here, $E$ is the electric field, IP is the ionization potential, $\Gamma$ is the mathematical gamma function, and $n=3.69 Z / \mathrm{IP}^{1 / 2}$ is the effective principal quantum number, with $Z$ the state of ionization, i.e. equal to 1 for a single ionized atom, $n_{e}$ is the electron density, and $n_{0}$ is the initial neutral density.

Thus the ionization rate of various ionic species of elements in our experiment can be obtained for different electric fields (E) using Eq. (15) and is plotted in Fig. 3(a). The peak rate of $10^{14} \mathrm{~s}^{-1}$ will result in full ionization for an electric field with a Gaussian profile and with $\sigma_{z}=40 \mu \mathrm{m}$. Therefore, from Fig. 3(a), $E_{0}$ of approximately $4 \mathrm{GV} / \mathrm{m}$ fully ionizes the first electron of the $\mathrm{Rb}$ atom $(\mathrm{Rb} \mathrm{I})$.

The $\mathrm{Rb}$ I ionization fraction contours for an electron beam with $\sigma_{z}=40 \mu \mathrm{m}$ and $\sigma_{r}=35 \mu \mathrm{m}$ are plotted in Fig. 3(b). It is clear that a portion of the beam's electrons in the front of the beam cannot ionize the $\mathrm{Rb}$ atoms since the beam's electric field up to this front is below the threshold value needed for ionization. These electrons, called the unaffected charge, therefore propagate through the $\mathrm{Rb}$ column with unmeasurable energy loss and appear at $20.35 \mathrm{GeV}$ on the spectrometer screen. The rest of the charge, which we call the participating charge will not only produce the plasma but also the wake and in doing so these electrons will lose some of their energy. Using the ADK model described above, we can define an ionization threshold as the location where $10 \%$ of the neutral atoms are ionized. It is then possible to estimate the amount of participating charge in the Gaussian profile by calculating the charge contained in the beam where the ionization threshold is exceeded. For a beam with $N=1.8 \times 10^{10}$, $\sigma_{z}=40 \mu \mathrm{m}$ and $\sigma_{r}=35 \mu \mathrm{m}$ (without any foils), the value of participating charge is $N^{*}=1.3 \times 10^{10}$ electrons, i.e. $72 \%$ of the initial charge. It is important to note that the peak current of the participating charge is the same as the original beam [red curve in Fig. 3(b) vs blue curve in Fig. 3(b)].

The bulk of the participating charge resides in the fully formed wake or the ion cavity where it experiences a linear transverse focusing force [2]. In other words, once the 


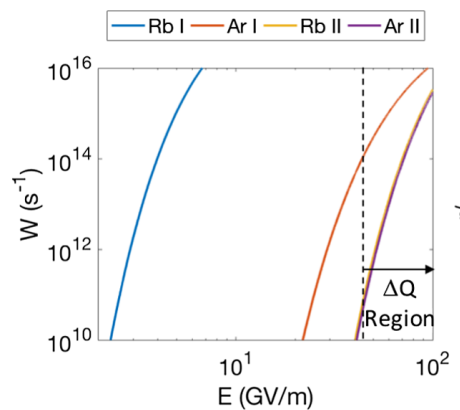

(a)

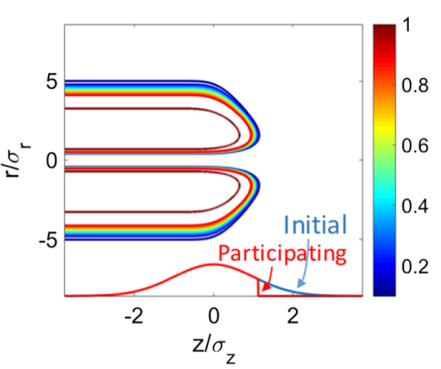

(b)

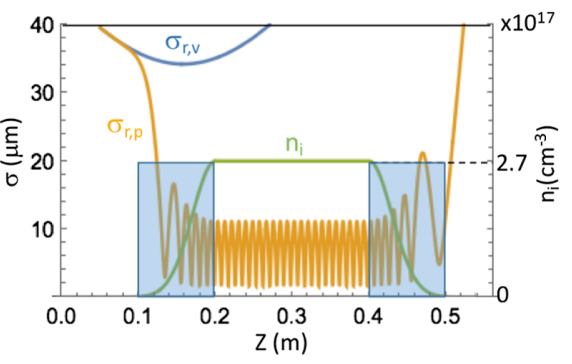

(c)

FIG. 3. (a) Ionization rates for neutral $\mathrm{Rb}$, neutral $\mathrm{Ar}, \mathrm{Rb}^{+}$, and $\mathrm{Ar}^{+}$ions as a function of electric field. (b) The ionization fraction for neutral $\mathrm{Rb}$ using an electron beam with $\sigma_{z}=40 \mu \mathrm{m}$ and $\sigma_{r}=35 \mu \mathrm{m}$. The rates are obtained from ADK model. The longitudinal profile of the initial beam is shown in blue lineout and the longitudinal profile of the participating charge is shown in red lineout. (c) Envelope evolution of a beam slice with normalized emittance of $\epsilon_{n}=250 \mathrm{~mm}$-mrad in an ion column with ion density $n_{i}$ having the same profile in $\mathrm{z}$ as the rubidium plasma. The plot of density is shown in green. The propagation of the electron beam in vacuum and in the presence of blowout regime is shown in blue and orange, respectively. Regions with significant presence of Ar are shown in blue shade. The beam size below $\sigma_{r}<7.9 \mu \mathrm{m}$ ionizes neutral $\mathrm{Ar}$ and $\sigma_{r}<4.5 \mu \mathrm{m}$ ionizes $\mathrm{Ar}^{1+}$ and $\mathrm{Rb}^{1+}$.

ionization threshold is exceeded, the beam electrons rapidly ionize the $\mathrm{Rb}$ atoms and blow out the plasma electrons. The evolution of the beam envelope in this ion column is described by [35]

$$
\begin{gathered}
\frac{d^{2} r}{d z^{2}}+k_{\beta}^{2} r=\frac{\epsilon_{n}^{2}}{\gamma_{b}^{2} r^{3}}, \\
k_{\beta}=\frac{\omega_{p}}{c} \frac{1}{\sqrt{2 \gamma_{b}}} .
\end{gathered}
$$

A beam with the transverse size equal to the matched spot size, given by $\sigma_{r \text {,matched }}^{2}=\epsilon_{n}\left(c / \omega_{p}\right) \sqrt{2 / \gamma_{b}}$, would maintain its transverse size while propagating in the plasma because its tendency to expand due to emittance is exactly balanced by the focusing force of the plasma ions. For our typical experimental parameters-taking $\epsilon_{n}$ as $250 \mathrm{~mm}$ $\operatorname{mrad}-\sigma_{r \text {,matched }} \sim 4 \mu \mathrm{m}$. Since the input beam radius in the experiment is larger than the matched spot size, the beam envelope's radius initially collapses as it enters the density up-ramp of the $\mathrm{Rb}$ column and then oscillates at half of the betatron wavelength $\left(\lambda_{\beta}=2 \pi / k_{\beta}\right)$ as shown in Fig. 3(c). In the uniform density region $\left(n_{p}=2.7 \times 10^{17} \mathrm{~cm}^{-3}\right)$ of our experiment, $\lambda_{\beta}=1.8 \mathrm{~cm}$. Note that the presence of the ramp causes a reduction in the amplitude of the envelope oscillation down to about $10 \mu \mathrm{m}$, which is less than half of $R_{b}$ as stated in Table I. This is important because the focusing force implicit in Eq. (17) applies to electrons within the blowout region $\left(r<R_{b}\right)$ only.

As the radius of the beam envelope decreases, the electric field of the beam increases according to Eq. (14), which results in an increase in the ionization rate according to Eq. (15). The different species can then be ionized inside the wake when the integrated ionization rate exceeds the threshold for their ionization. These species are the second electron of rubidium $\left(\mathrm{Rb}^{1+}\right.$ to $\left.\mathrm{Rb}^{2+}\right)$ and the first and second electrons of argon buffer gas in the density up and down ramps. Using the current profile shown in Fig. 3(b), the radius at which the ionization fraction of various species would surpass a threshold of $10 \%$ can be calculated. For instance, neutral $\mathrm{Ar}$ is ionized when $\sigma_{r}<7.9 \mu \mathrm{m}$ and $\mathrm{Ar}^{1+}$ and $\mathrm{Rb}^{1+}$ are ionized when $\sigma_{r}<4.5 \mu \mathrm{m}$. These electrons are ionized within the wake, gain energy from the wakefield, and can accumulate at the rear of the wake [18] as the electron drive beam executes more and more betatron oscillations.

Argon electrons are ionized and trapped in the ramp region only. The electrons resulting from ionization of $\mathrm{Rb}^{1+}$ on the other hand are ionized and captured at every cycle of the betatron oscillations, and with the betatron wavelength of $1.8 \mathrm{~cm}$, this represents about 22 discrete regions (there are two envelope oscillations per betatron wavelength, which is defined for the oscillatory motion of a single electron in the ion column) along the density plateau of the plasma when $\sigma_{r}$ goes below $4.5 \mu \mathrm{m}$. This means that unlike Ar electrons, $\mathrm{Rb}$ II electrons can be generated and injected in a distributed fashion along the length of the beam-plasma interaction. Therefore, in this scenario, the injection of Ar electrons is localized to the ramp regions, whereas the amount of the injected Rb electrons is proportional to the interaction length.

One way to circumvent the ionization and injection of this secondary charge in future experiments is to use a large matched beam. This could be done by increasing the emittance or reducing the plasma density or by a combination of the two.

In addition to causing secondary ionization of $\mathrm{Rb}$, the betatron oscillation of the electrons in the blowout region generates $\mathrm{X}$-ray radiation because of the transverse acceleration of the electrons due to the linear focusing force of the ion column. Most of the radiation is emitted at the extrema of the electron orbit where the velocity is zero 
and the acceleration is maximum. The average energy radiated by a single electron in the blowout region can be calculated from [36]

$$
W_{s}=\bar{P}_{s} \times t=\left(\frac{e^{2} c^{2}}{12} \gamma_{b}^{2} k_{p}^{4} r_{0}^{2}\right)\left(\frac{N_{\beta} \lambda_{\beta}}{c}\right),
$$

where the first factor represents the average power generated by an electron in a betatron cycle, and the second factor represents the interaction time. In this expression, $N_{\beta}$ is the number of betatron oscillations, $\gamma_{b}$ represents the energy of the participating charge, and $r_{0}$ is the amplitude of an electron's oscillation in the blowout region. In other words, for a given plasma density and beam energy, the amount of radiation depends on the interaction length, $N_{\beta} \lambda_{\beta}$, and the radius of oscillation in the blowout region, $r_{0}$.

The insertion of foils F1-F3 affects the measurable quantities in the experiment through its effect on the emittance and the transverse size of the electron beam. By increasing the beam emittance (see Table II), the insertion of a foil results in higher head erosion rate and therefore shorter plasma length. This results in a reduction in the value of all measurable quantities. Note that the increase in emittance here is not sufficient to match the beam to the plasma.

The effect of the increased emittance is compounded by an increase in $\sigma_{r}$, also shown in Table II. The increased $\sigma_{r}$ results in lower radial electric field of the beam according to Eq. (14), which affects the experiment in three ways.

First, the reduced electric field results in a reduction in the fractional ionization of neutral $\mathrm{Rb}$ as the electron beam enters the plasma. The initial rubidium plasma forms farther away from the $r=0$ axis, and simulations show that this effect results in a delay in the formation of the blowout regime. Similar to the head erosion effect, this hollow plasma formation thus contributes to a reduction in the interaction length.

Second, the increased $\sigma_{r}$ and the delay in the generation of the blowout regime mean that the beam slices may not experience the effect of the ramp. Therefore, the peak oscillation amplitude of the beam radii for various beam slices may not reduce below the value of $R_{b}$. This results in reduction of participating charge in each slice. The reduced participating charge per slice results in a proportional reduction in the $\mathrm{x}$-ray signal as fewer electrons perform betatron oscillations. In the experiment, we accounted for the reduced participating charge by normalizing the total $\mathrm{x}$-ray signal count by the amount of participating charge in each shot. The participating charge was measured for each data point by measuring the charge outside energies of 19.9 and $21.2 \mathrm{GeV}$. Additionally, the reduced participating charge per slice in the blowout region can be interpreted as reduced current, and since the head erosion rate depends strongly on the current, the increased $\sigma_{r}$ implies a shorter plasma.
Third, the increased $\sigma_{r}$ results in an ionization front that is closer to the center of the beam; i.e. closer to $z=0$ in Fig. 3(b). This backward motion of the ionization front results in further reduction in the interaction length due to having an effectively shorter $\sigma_{z}$ for head erosion.

Through the mechanisms described above, the insertion of each foil is expected to strongly reduce the interaction length incrementally from foil $1-3$.

\section{EVIDENCE FOR DISTRIBUTED INJECTION}

The evidence for the distributed injection of electrons throughout the interaction length comes from the examination of the excess charge $\Delta Q$ with respect to other parameters that depend on length. The two parameters that depend strongly on length and are therefore used here for its assessment are the x-ray yield and the energy loss. It is important to study the variation of both, because even though both share a dependence on the interaction length, the energy loss is dependent on the longitudinal profile of the beam ( $\Lambda$, as described above), but the $\mathrm{x}$-ray yield is dependent on the transverse dynamics of the beam. Therefore, a correlation of both energy loss and x-ray yield is an indication of a change in the interaction length, which is the common parameter between the two. The interaction length in this experiment was varied by either changing the beam current as measured by the pyro signal or by changing the beam emittance, which was achieved by inserting one of the foils F1-F3 in the beam path.

The effect of beam current on the experiment can be observed from one data set with 77 shots (Fig. 4), where the change in $\sigma_{z}$ came about due to random phase variations in the linac. This set of data was chosen because of relatively wide variation in various quantities, which allows for meaningful correlation of various parameters in the experiment: pyro signal varies from 3500 (representing $\sigma_{z} \sim 48 \mu \mathrm{m}$ ) to 5500 (representing $\sigma_{z} \sim 39 \mu \mathrm{m}$ ), $\Delta Q$ varies from 100 to $700 \mathrm{pC}, \Delta W^{-}$from 3.5 to $5.5 \mathrm{GeV}$, and x-ray yield varies by nearly a factor of 2 . Larger $\sigma_{z}$ corresponds to lower current, and since the head erosion rate depends on $I^{-3 / 2}[5,7]$, the reduced beam current is expected to lead to higher head erosion rate, and decreased interaction length.

The $\mathrm{x}$-ray yield in this data set, i.e. integrated $\mathrm{x}$-ray signal per electron, increases by a factor 1.9 for increasing values of current (lower $\sigma_{z}$ ) as shown in Fig. 4(a). Other parameters besides interaction length responsible for this variation [see Eq. (19)] include plasma density, which is constant for this data, $\gamma_{b}$, and the transverse oscillation radius, $r_{0}$. The variation in $\gamma_{b}$ attributable to the range of $\Delta W^{-}$is on the order of $10 \%$ ( 29000-33000), which implies a corresponding change in $\mathrm{x}$ rays on the order of $30 \%$. Assuming relatively small changes in transverse radius, the reduction in interaction length, i.e. $N_{\beta} \lambda_{\beta}$, accounts for the rest of the difference in X-ray yield. In other words, the length of the interaction was reduced by up to $63 \%$. 

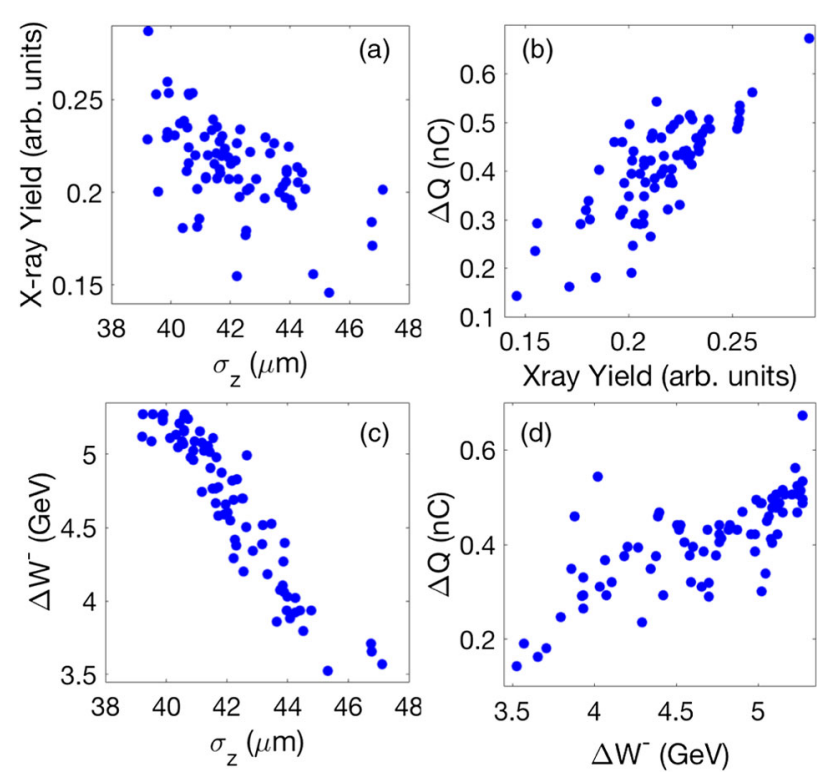

FIG. 4. (a) X-ray yield for a set of 77 consecutive shots sorted as a function of $\sigma_{z}$. In this data set, foil F1 is inserted and the variation of $\sigma_{z}$ is due to the shot-to-shot jitter of the phase of the linac. (b) $\Delta Q$ and $\mathrm{x}$-ray yield are plotted against each other for each shot. (c) $\Delta W^{-}$sorted as a function of $\sigma_{z}$ for the same data. (d) $\Delta Q$ correlation with $\Delta W^{-}$for the same data.

The correlation between $\Delta Q$ and the $\mathrm{x}$-ray yield [Fig. 4(b)] is nearly linear, which suggests that $\mathrm{x}$ rays and $\Delta Q$ electrons are generated in proportion as expected based on the physical picture described above, i.e. both are associated with the spot size oscillation during betatron cycles of the beam in the ion column and therefore their yield increases as the plasma length increases. It is also important to emphasize here that the oscillations of the different longitudinal slices of the beam are not in phase, as will be shown in the simulation section. This means that $\mathrm{x}$ rays and $\Delta Q$ do not increase with length in discrete steps of $\lambda_{\beta}$ and therefore the relationship between the two shows a continuous curve.

A second correlation between the beam current and the maximum energy loss of the beam electrons $\Delta W^{-}$[Fig. 4(c)] further implies that the reduction of interaction length (and therefore $\Delta W^{-}$) is due to the reduction of beam current for the data shown in Fig. 4. As previously mentioned, $\Delta W^{-}$ depends on the decelerating field $E^{-}$and the interaction length and is independent of the transverse dynamics of the beam which determines both the x-ray yield and $\Delta Q$. Therefore, the linear correlation between $\Delta Q$ and $\Delta W^{-}$ [Fig. 4(d)] is important as it underscores the fact that the change in $\Delta Q, \Delta W^{-}$and $\mathrm{x}$-ray yield are all caused by variations in interaction length and further strengthens the argument for the distributed injection of electrons.

The second way to reduce the propagation length is by increasing the beam emittance by inserting one of foils (F1-F3) in the beam path as discussed earlier. Since each foil contributes to progressively larger emittance it is expected that the interaction length decreases with a higher-numbered foil.

Once again, the reduced interaction length can be inferred by examining the x-ray yield and $\Delta W^{-}$. By solving the beam envelope equations [Eq. (17)], one can show that the amplitude of the betatron oscillations would grow by a factor of 3 as a result of the increased spot size at the entrance of the plasma [24,37]. The minima of the oscillation on the other hand, remains nearly the same. In other words, had the beam propagation length remained unchanged, a significant increase (about a factor of 9) in $\mathrm{x}$-ray yield per electron would have been observed due to much larger oscillation amplitude $\left(r_{0}\right)$ as a result of inserting a foil. In the experiment however, insertion of a foil led to a significant decrease in the $\mathrm{x}$-ray yield, as

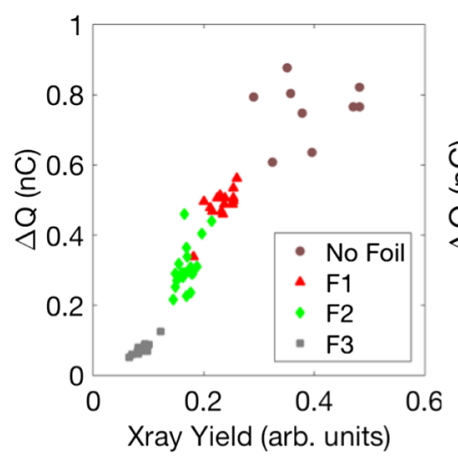

(a)

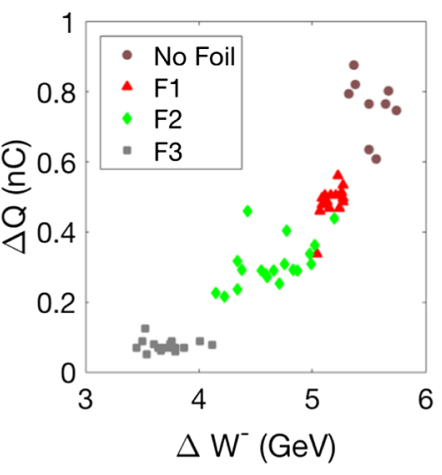

(b)

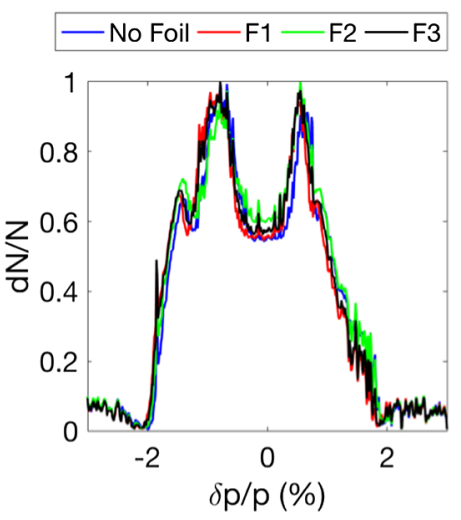

(c)

FIG. 5. (a) Excess charge as a function of the x-ray yield in four cases obtained with the insertion of various foils that change the beam emittance. The data is filtered by selecting pyro values between 5000 and 5300, $\left(\sigma_{z} \sim 40 \mu \mathrm{m}\right)$ so that the current profiles are nearly identical as judged by the similarity of the electron beam spectra before the plasma. The data shown in red triangles is a subset of data in Fig. 4. (b) Reproduced from [38]; energy loss is shown as a function of excess charge for the same data as in (a). (c) The electron beam energy spectra for the data shown in (a) and (b). The spectra related to each foil is averaged. 
shown in Fig. 5(a). The data in this figure is selected such that the beam current is the same for the data points. In this case, the value of pyro chosen implies a beam with $\sigma_{z} \sim 39 \mu \mathrm{m}$. The similarity in the longitudinal profile of these data points is confirmed by inspecting the beam energy spectrum, which is shown in Fig. 5(c).

In addition to $\mathrm{x}$-ray yield, $\Delta Q$ also decreases significantly for each increasing number of the foil [Fig. 5(a)]. Once again, this correlation is consistent with a reduced beam propagation distance in the plasma as the beam emittance is increased. To corroborate the dominant role of reduction of the interaction length as the cause of the decrease in both $\Delta Q$ and $\mathrm{x}$-ray yield, $\Delta Q$ is plotted as a function of $\Delta W^{-}$for the same data in Fig. 5(b). It is observed that $\Delta W^{-}$, which depends on $E^{-}$and interaction length rather than $\sigma_{r}$ and the transverse dynamics of the beam, monotonically increases with higher $\Delta Q$. Once again, this result shows that the longer the interaction length, (i.e. the higher the $\Delta W^{-}$), the higher the number of excess electrons that are generated and injected into the wake, and so the source of electrons comprising $\Delta Q$ is likely distributed over the interaction length. Since the linear relationship between the charge and length is a characteristic of the electrons ionized and trapped from rubidium and not argon (which is localized at the either ends of the Rb column), the source of the increase in the dark current is consistent with being comprised mainly of the $\mathrm{Rb}$ II electrons.

\section{BEAM LOADING DUE TO DISTRIBUTED INJECTION}

The $\mathrm{Rb}$ II electrons ionized along the length of the interaction can accumulate at the back of the wake, thereby beam loading the wake and reducing the accelerating field. This effect was observed in the experiment as reduced average transformer ratio. The transformer ratio is defined as the ratio of the accelerating field to decelerating field $\left(R=E^{+} / E^{-}\right)$. But since the electric fields were not directly measured in the experiment, the energy loss and energy gain are used instead to define an average transformer ratio $\langle R\rangle=\Delta W^{+} / \Delta W^{-}$in order to examine the effect of distributed injection of $\mathrm{Rb}$ II electrons on the fields in the experiment.

To illustrate the beam loading effect of the $\mathrm{Rb}$ II electrons, energy loss and gain for another data set with 71 consecutive shots are examined as shown in Fig. 6. This particular data set is of interest because $\Delta W^{-}$for most of the data is confined to
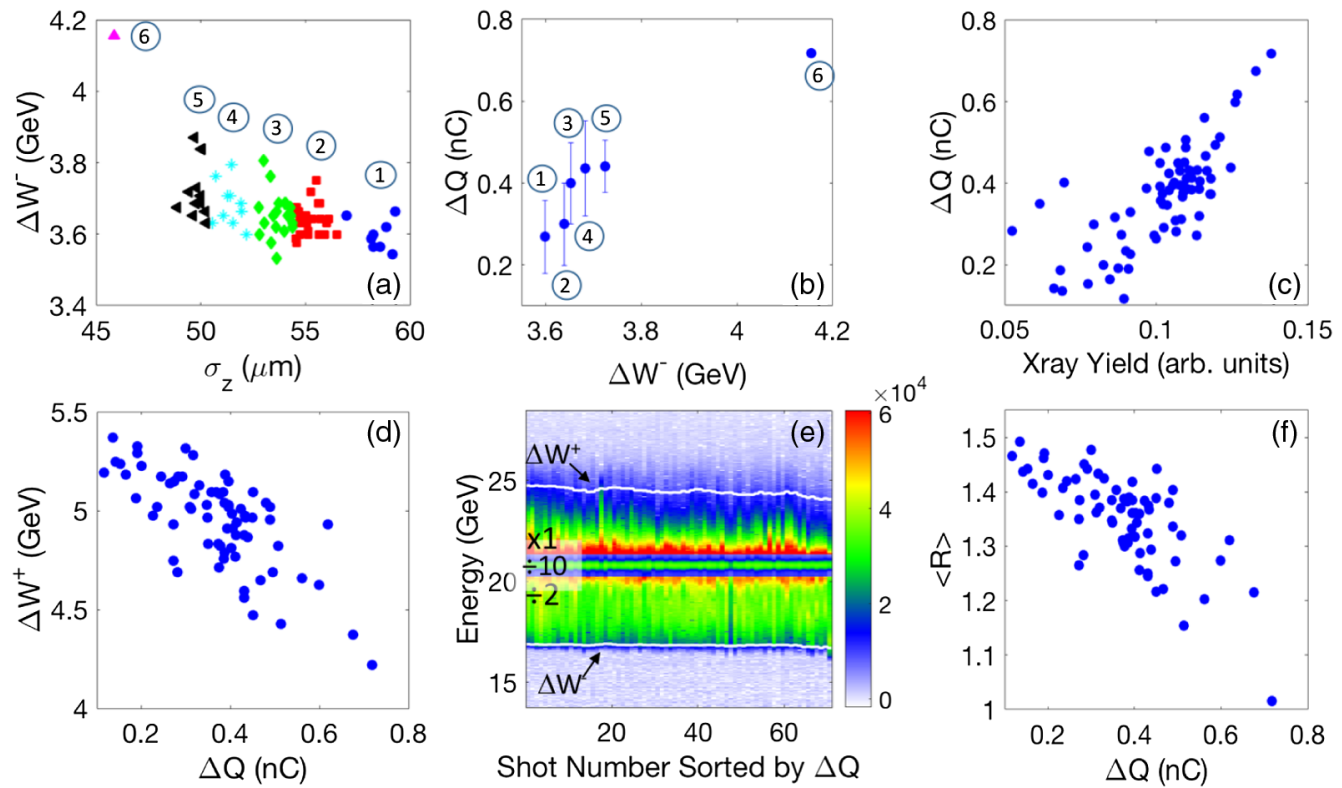

FIG. 6. Data from a 71 shot data set where no foils F1-F3 were inserted. (a) Peak energy loss $\Delta W^{-}$as a function of $\sigma_{z}$, grouped by a range of 300 in pyro corresponding to $\sim 3 \mu \mathrm{m}$ change in $\sigma_{z}$. (b) $\Delta Q$ plotted as a function of $\Delta W^{-}$, where each point corresponds to one of the six groups in (a). The corresponding groups in (a) and (b) are indicted by numbers above or below data points. The value of $\Delta W^{-}$for each data point in (b) corresponds to the mean value of $\Delta W^{-}$for a group in (a) and the mean of $\Delta Q$ for each group is plotted with the error bars representing the standard deviation of $\Delta Q$ in a group. (c) Correlation of x-ray yield and $\Delta Q$ data shown in (a). (d) Peak energy gain $\Delta W^{+}$as a function of $\Delta Q$ for the same data. $1 \mathrm{GeV}$ is added to the raw $\Delta W^{+}$value to compensate for the head-to-tail energy chirp of the incoming electron beam. (e) Waterfall plot for data shown in (a). Each vertical slice represents the energy spectra for a shot, which is obtained by integrating the spectra along the nondispersive direction. The shot number is shown on the horizontal axis, and the data is sorted by increasing values of $\Delta Q$ along this axis. The counts near the initial beam energy $(\sim 20.35 \mathrm{GeV})$ and the counts below the initial energy (energy loss area) are divided by 10 and 2, respectively, and as indicated on the images. (f) Average transformer ratio, $\langle R\rangle=\Delta W^{+} / \Delta W^{-}$plotted for the same data set shown in (e). 
a small range of values between 3.5 and $3.8 \mathrm{GeV}$ [Fig. 6(a)]. Therefore, the reduction of the average transformer ratio due to the beam loading of the excess charge can be clearly attributed to the beam loading of the accelerating field. It is important to note that as discussed in the previous section, $\Delta W^{-}$increases with increasing current (i.e. decreasing $\sigma_{z}$ ), as shown in Fig. 6(a).

Even though the general trend of the peak energy loss increasing with $\sigma_{z}$ is observed in this data set [Fig. 6(a)], there is a large variability in the energy loss in each group. The spread in this data set is attributed to the effect of the transverse size of the beam on the integrated pyroelectric signal. In general, the correlation of the integrated signal from the pyroelectric detector with $\sigma_{z}$ is correct as observed in Fig. 6(a). Within a small range of variation of the pyro however, the changes in the transverse size of the beam can affect the signal observed by the detector. To remove this effect from the interpretation of the $\Delta W^{-}$data, the data points within a small range of pyro (corresponding $\pm 1.5 \mu \mathrm{m}$ in $\sigma_{z}$ ) are grouped together as shown in Fig. 6(a).

Removing the spread of the data in the energy loss makes the correlation with $\Delta Q$ more observable. This was done in Fig. 6(b), where the mean and standard deviation of the $\Delta Q$ is plotted for the mean of $\Delta W^{-}$within each group identified in Fig. 6(a). Although the change in the interaction length is expected to be fairly small, even within this small change, one can observe the correlation of the $\Delta Q$ with energy loss as evidence of distributed injection as explained earlier. Note that if the range of pyro is extended by adding data from other data sets such that the overall change in pyro is dominated by beam current and the range of $\Delta W^{-}$is extended, the same correlation between $\Delta Q$ and $\Delta W^{-}$as described earlier is clearly observed [38].

The relative small change in $\Delta W^{-}$implies that the peak current and the interaction length are fairly constant. On the other hand, both $\Delta Q$ and $\mathrm{x}$-ray yield show several folds of variability and are again strongly correlated [Fig. 6(c)], further supporting our model, which asserted that over every cycle of $\mathrm{x}$-ray generation, excess charge is produced, i.e. injection occurs in a distributed fashion. Therefore, in this scenario, we can examine the effect of the accumulated charge on the energy gain directly for a relatively constant length.

As $\Delta Q$ increases from $100 \mathrm{pC}$ to over $700 \mathrm{pC}$, the $\Delta W^{+}$ decreases by over $1 \mathrm{GeV}$ [Fig. 6(d)]. Note that this variation is about 5 times higher than the variation in $\Delta W^{-}$, and that this change is very strongly correlated with the change in excess charge. Using Eq. (4) in Sec. II, one can observe that this $\Delta Q$ is enough to reduce the accelerating field by half.

Correspondingly, the value of $\langle R\rangle$ decreases from 1.5 to about 1 [Fig. 6(f)]. This effect is visually illustrated in Fig. 6(e), where data is sorted according to how much $\Delta Q$ was generated during each shot. The energy gain can be clearly seen as decreasing while the energy loss stays more or less the same. It is important to note that, while this

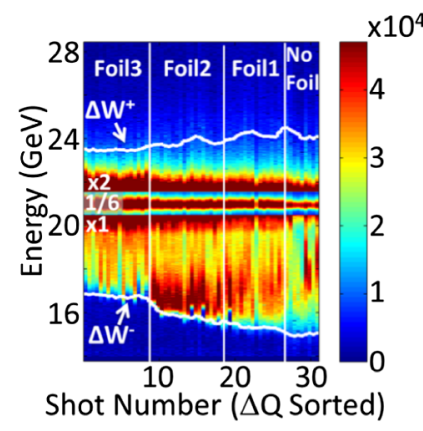

(a)

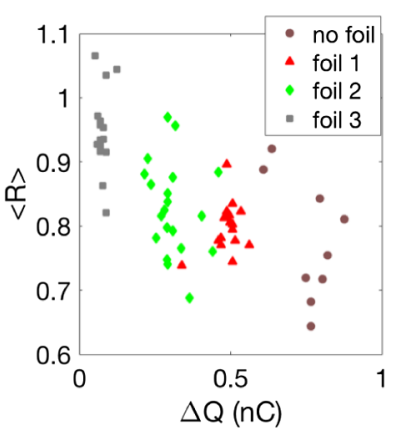

(b)
FIG. 7. Originally appeared in [38]. (a) Measured energy spectra for the same shots as displayed in Fig. 5. The white line shows $\Delta W^{-}$and $\Delta W^{+}$, where each point represents moving average of five shots. (b) The measured average transformer ratio $\langle R\rangle=\Delta W^{+} / \Delta W^{-}$as a function of $\Delta Q$.

reduction in the average transformer ratio is accompanied by variation in the longitudinal beam size inherent in the shot to shot variations of the linac, this effect is not important to calculations of $\langle R\rangle$. As will be seen later in the simulation portion of this paper, the injected electrons accumulate at a certain phase in the wake, where the accelerating field becomes significantly suppressed. The accelerating gradient experienced by electrons behind that phase in the wake will be suppressed due to beam loading and therefore those electrons do not contribute to the $2 \%$ of the charge that is used to determine the energy gain.

The effect of beam loading can also be observed when the longitudinal profile is carefully controlled as well. For instance, taking the same profiles used to make Fig. 5, it is possible to observe increased beam loading and suppression of transformer ratio in Fig. 7. In this case, the energy loss increased due to the increased interaction length as lower numbered foils are inserted in the beam path as discussed earlier. As the energy loss is increased, the energy gain increases as well, but it does so at a slower rate because the $\Delta Q$ (accumulated up to $\sim 1 \mathrm{nC}$ ) heavily beam loads the wake. In other words, increasing excess charge $(\Delta Q)$ leads to a reduction of the transformer ratio.

\section{SIMULATIONS}

2D cylindrical symmetric OSIRIS PIC code [39] was used to simulate the interaction of an electron beam with a rubidium plasma. The simulation confirms the physical picture of beam loading by distributed injection described above. The results show that both argon atoms and rubidium ions are ionized inside the wake as a result of reduction of the beam radius in a betatron cycle, and as a result both contribute to $\Delta Q$. Additionally, the simulations show that $\Delta Q$ causes significant beam loading.

The plasma density is modeled as a trapezoid with a $20 \mathrm{~cm}$ plateau region at a density of $2.7 \times 10^{17} \mathrm{~cm}^{-3}$ and $10 \mathrm{~cm}$ ramps on either side that reduce the density to zero. 


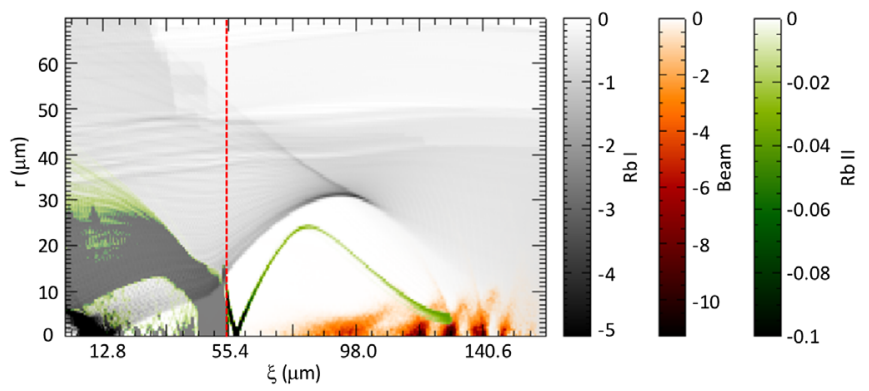

FIG. 8. A density plot of a snapshot in the simulation. All densities are normalized to $2.7 \times 10^{17} \mathrm{~cm}^{-3}$. Rb II electrons in the area to the left of the red dashed line are made transparent, so that the deflection of the sheath due to the beam loading of excess charge is visible. Since the simulation is cylindrically symmetric, only $r>0$ is shown in this image. Rb I electrons form the wake and are shown in gray scale while Rb II electrons, shown in green, are tracked separately from $\mathrm{Rb}$ I electrons. The color plots are restricted for better visibility of lower density features.

The simulation box has $480 \times 400$ square cells of $0.05 k_{p}^{-1}$ wide, where $k_{p}^{-1}$ is the plasma skin depth. A speed-of-light moving window is used in the direction of the beam's propagation. The ionization model used in the simulation was the ADK formalism [Eq. (15)] and ionization of Rb, $\mathrm{Rb}^{+}, \mathrm{Ar}$, and $\mathrm{Ar}^{+}$were included, with eight particles per cell allocated to $\mathrm{Ar}$ and $\mathrm{Ar}^{+}$while four particles per cell were allocated to $\mathrm{Rb}$ and 16 allocated to $\mathrm{Rb}^{+}$ions. $\mathrm{Rb}$ and $\mathrm{Rb}^{+}$ions are treated as separate species, so that the ionization injection and self-injection events can be separated. The electron beam is allocated four particles per cell and contains $1.8 \times 10^{10}$ electrons with $\sigma_{z}=35 \mu \mathrm{m}, \epsilon_{n}=$ $250 \mathrm{~mm}-\mathrm{mrad}$ and is focused to a waist of $\sigma_{r}=35 \mu \mathrm{m}$. These parameters represent the experimental case where the electron beam current was at its highest value and no foils have been inserted, and therefore the electron beam would propagate the farthest in the plasma.

A snapshot of the simulation, shown in Fig. 8, illustrates secondary ionization and injection of Rb II electrons. In this snapshot, the electron beam has already propagated $7 \mathrm{~cm}$ into the plasma (i.e. still within the ramp), its transverse radius has already been reduced from the original value of $35 \mu \mathrm{m}$, and its envelope is executing betatron oscillations. Several important characteristics of the experiment can be observed from this figure.

First, it can be observed that various longitudinal slices of the beam perform envelope oscillations at different phases with respect to each other. The slices where the envelope radius is at its lowest value [the valley in Fig. 3(c)] produce an increased beam electron density (darker red), while those slices with the highest radius (peak of their oscillation) produce lower density (lighter red). The reason for the phase variation in the longitudinal slices is the blowout distance, which is given by $\frac{2}{k_{p}} \sqrt{\frac{n_{p}}{n_{b}}} \sim 2 c / \omega_{p}$ [5]. The electron density continuously decreases down to zero (blowout) over this distance, which means the various longitudinal slices over this distance see different plasma density. Additionally, the longitudinal field structure results in different energies for the various longitudinal slices. Since the transverse dynamics of the beam [11] and particularly the betatron wavelength [Eq. (18)] depend on density and energy, different slices of the beam will come to perform their betatron oscillations at different phases with respect to each other.

Second, $\mathrm{Rb}$ II electrons (shown in green color scale) are generated inside the wake at a transverse distance approximately equal to the radius of a beam slice at the pinch point of its oscillation cycle. Rb II electrons ionized earlier in the simulation can be observed moving within the wake (thin green line). Since different slices reach their minimum size at different times, and hence different distances along the interaction length, $\mathrm{Rb}$ II injection takes place continuously along the length of the interaction rather than in a periodic manner, which would have been expected if all slices performed their betatron oscillations in phase.

Third, the trajectory of the newly ionized Rb II electrons can be easily inferred from Fig. 8. These electrons move to the back of the wake and start accumulating there. The onaxis density of these electrons, which occupy a very small space, reaches as high as $8.1 \times 10^{19} \mathrm{~cm}^{-3}, 300$ times greater than the initial neutral density, which in turn leads to a tremendous transverse space charge field, peaking at over $460 \mathrm{GeV} / \mathrm{m}$. This radial electric field ionized a vertical sheet of $\mathrm{Rb}$ II electrons (green), which are then immediately pushed out even through the sheath of the blowout region (grey) that is composed of Rb I electrons. Note that in this simulation, only the first two ionization levels of $\mathrm{Rb}$ atom were included. However, had the higher ionization levels of $\mathrm{Rb}$ been included in the simulation, some would likely be ionized as well and likely reduce the value of this field.

Although argon electrons (not shown in Fig. 8) are generated in the same way, their effect is localized only to the ramps and therefore, the number of argon electrons trapped is lower than Rb II electrons. In this simulation, the maximum number of trapped argon charge, measured as charge with $\gamma_{b}>200$ was $100 \mathrm{pC}$, reached at $10 \mathrm{~cm}$ - or at the top of the ramp. From that point, the number of argon electrons slowly decreased, such that by $15 \mathrm{~cm}$, only $30 \mathrm{pC}$ of these argon electrons remained within the wake. In comparison, Rb II electrons with $\gamma_{b}>200$ monotonically increased to $350 \mathrm{pC}$ at $15 \mathrm{~cm}$. In other words, after the ramp, $\mathrm{Rb}$ electrons kept being trapped while Ar electrons left the simulation box.

Finally, the beam loading of the wakefield by the injected charge can be observed in Fig. 8 in the form of the sheath crossing point being pushed back in the frame of the beam. This is expected because the longitudinal field of the wake is related to the slope of the sheath [4,12], and as the injected electrons beam load the wake, they absorb the 

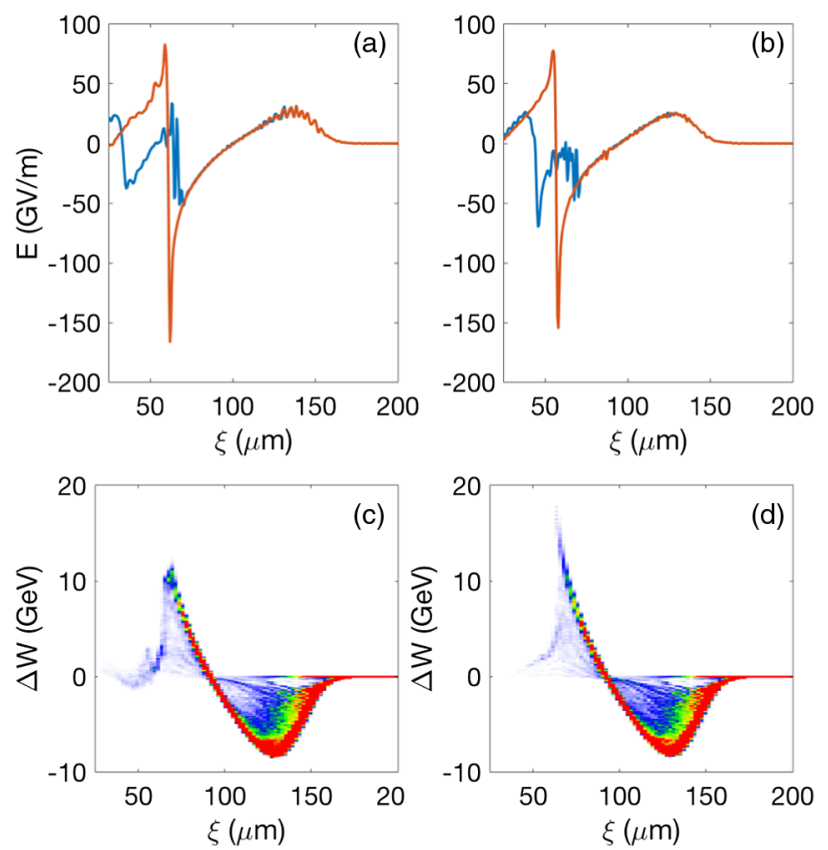

FIG. 9. Comparison of simulations with and without $\mathrm{Ar}$ and secondary $\mathrm{Rb}$ ionization. (a) On-axis electric field for the two simulations with the beam having propagated $12 \mathrm{~cm}$ in the plasma in each simulation. The red curve represents the value of the field where only ionization of $\mathrm{Rb} \mathrm{I}$ is present. For the blue curve both ionization levels of $\mathrm{Ar}$ and $\mathrm{Rb}$ are included. (b) Same parameters plotted as (a), except that the frame represents a time at $30 \mathrm{~cm}$ of beam propagation. (c) The energy space of the beam at the end of the simulation for the beam loaded case. Peak energy loss of $8 \mathrm{GeV}$ and energy gain of 12 are clearly observed. Part (d) is the same for the simulation without beam loadingi.e. secondary ionization injection.

electric field of the wake and reduce it. Thus, this beam loading results in a modification of the slope of the sheath, and the significantly reduced slope at the site of the injected electrons can be interpreted as a clear indication of significant beam loading.

The beam loading of the injected charge can be quantified by investigating the longitudinal accelerating field as illustrated in Fig. 9. Two simulation times are shown in this figure, one where beam has propagated $12 \mathrm{~cm}$, which is $1 \mathrm{~cm}$ after the beam has entered the plateau, and the second, where the beam has traveled $30 \mathrm{~cm}, 1 \mathrm{~cm}$ before the beam enters the down ramp. To emphasize the effect of beam loading, another simulation was performed, which is identical to the first, except that the secondary ionizations are turned off; in other words, the only ionization state allowed is the ionization of $\mathrm{Rb}$ I. The on-axis electric fields in the simulation with and without secondary ionization are shown in blue and red, respectively.

Comparing the two simulations at $12 \mathrm{~cm}$, it can be observed that the accumulated $\mathrm{Ar}$ and $\mathrm{Rb}$ II electrons have already reduced the peak electric field from about $82 \mathrm{GV} / \mathrm{m}$ in the unloaded case to about $46 \mathrm{GeV}$; a drop of
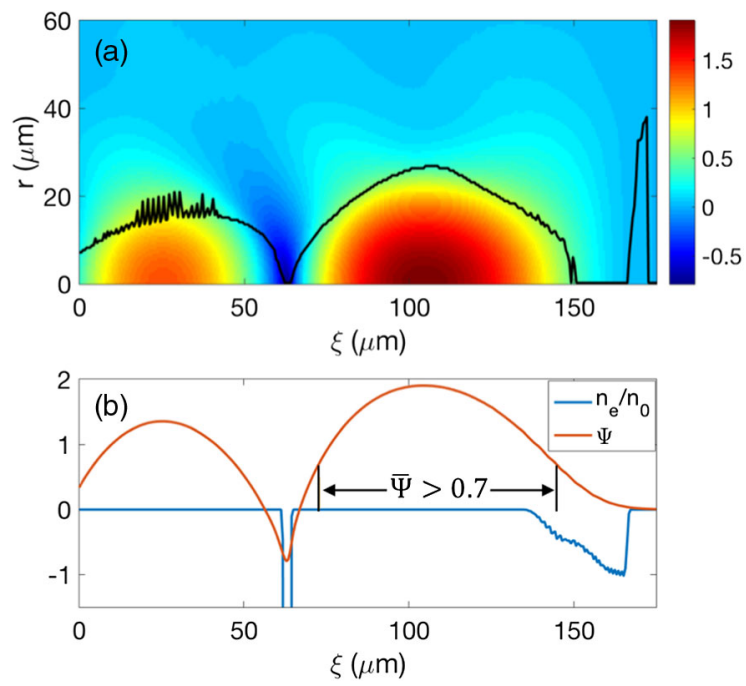

FIG. 10. (a) $\bar{\Psi}$ for the simulation frame shown in Fig. 9(a) for the case where no secondary ionization is present. The black curve shows the outline of radial location, where $\mathrm{Rb}$ I electron density corresponds to $50 \%$ neutral density, i.e. the bubble sheath for $\xi<160 \mu \mathrm{m}$. The spike in the region $165<\xi<170 \mu \mathrm{m}$ indicates the region over which the neutral $\mathrm{Rb}$ is ionized. (b) The outline of on-axis plasma density (blue) and $\bar{\Psi}$ (shown in orange) corresponding to the $r=0$ line in (a). On-axis density reaches 1 (indicating full ionization on axis) at $\xi=165 \mu \mathrm{m}$. In the region $73.0 \mu \mathrm{m}<\xi<144.8 \mu \mathrm{m}, \bar{\Psi}>0.7$. Electrons born in this region that reach the back of the wake where $\bar{\Psi} \sim-0.3$ satisfy the trapping condition $\Delta \bar{\Psi}<-1$.

nearly a factor of 2 . At this point, with peak decelerating field being $32 \mathrm{GV} / \mathrm{m}$, the transformer ratio at this point is reduced from $\sim 2.7$ in the unloaded case to 1.6 in the beam loaded case.

Between 12 and $30 \mathrm{~cm}$, the beam loading region widens as more charge is added. It can be observed that after $30 \mathrm{~cm}$ of propagation, the accelerating field is reduced almost to zero, where it would have been at its maximum in the unloaded (red lineout) case. As a result of this beam loading, the sheath crossing is pushed backwards resulting in the spike of the accelerating field to be moved from the original $\xi=58 \mu \mathrm{m}$ to $\xi=45 \mu \mathrm{m}$ [Fig. 9(b)]. It is also noteworthy that the decelerating parts of the wakes are nearly identical with and without secondary ionizations as expected because of causality.

Additionally, there is no excess charge observed in the simulation without secondary ionization. This can be explained by inspecting the values of $\bar{\Psi}$, which are shown for the same condition as Fig. 9(a) in Fig. 10. In Fig. 10(a) the $\bar{\Psi}$ function is shown as a color map and the outline of the wake's sheath is overlaid on the color map as a black line. The location of the sheath is important because the focusing force of the ion column exists only in the region bounded by it. In particular, it is important for the trapping condition to be satisfied before the high density spike at the back of the wake, which is $\sim 70 \mu \mathrm{m}$ on axis [see Fig. 10(b)]. 
This is because the high density spike at the back of the wake has a very high transverse field, and if the electrons are trapped before they reach this spike, they would get ejected transversely out of the wake region at this location.

As discussed in the Sec. III, an electron that will have $\Delta \bar{\Psi}<-1$ can get trapped in the wake. Since the value of the $\bar{\Psi}_{f}$ at the back of the wake (just ahead of the density spike) is $\sim-0.3$ [see Fig. 10(b)], the electrons born outside the wake will have a maximum $\Delta \bar{\Psi}$ of -0.3 . This value of $\Delta \bar{\Psi}$ is too low to allow for trapping and therefore background plasma electrons are not trapped by the wake. The electrons born inside the wake on the other hand, can have a $\bar{\Psi}_{i}$ of up to 1.9 depending on where they are born. Because $\bar{\Psi}_{f} \sim-0.3$, those electrons with $\bar{\Psi}_{i}>0.7$ will be able to satisfy the trapping condition $\Delta \Psi<-1$. This region of $\bar{\Psi}_{i}>0.7$ is indicated in Fig. 10(b). Therefore, the Rb I electrons that are ionized early by the beam do not get trapped in the simulation, and the excess charge is present only due to the ionization injection of electrons from $\mathrm{Ar}$ and $\mathrm{Rb}^{1+}$ ions.

The final energy space of the beam in loaded and unloaded simulations [Figs. 9(c) and 9(d)] shows how the energy gain and loss are affected by beam loading. This figure is important because energy gain $\left(\Delta W^{+}\right)$and energy loss $\left(\Delta W^{-}\right)$have observable counterparts in the experiment. In the simulation without beam loading [Fig. 9(d)], the average transformer ratio $\langle R\rangle=\Delta W^{+} / \Delta W^{-}=17.5 / 8=2.2$, whereas in the beam loaded case, $\langle R\rangle=12 / 8=1.5$. In the experiment, the $\langle R\rangle$ varies from 1.5 to 0.75 depending on the excess charge (see Figs. 6 and 7). The smaller $\langle R\rangle$ is to be expected in the experiment because in the experiment, the tail electrons in the drive bunch apparently do not sample the maximum electric field of the wake.

We note that although nearly $0.8 \mathrm{nC}$ of RbII electrons were injected in the simulation, i.e. with $\gamma_{b}>200$, not all these electrons are necessarily being accelerated at the location of the initial accelerating spike and thus do not contribute to beam loading at that location. Of the total $\Delta Q$ of up to $1 \mathrm{nC}$, only about $300 \mathrm{pC}$ of $\mathrm{Rb}$ II electrons are injected and accelerated at the site of the initial spike and the rest of the secondary injection occurred at the second spike, which was generated due to the elongation of the back of the wake. Only the former contribute to the beam loading and the reduction of $R$. This latter amount is consistent with the estimate of the charge that would reduce the accelerating field by half from Sec. II. It is further interesting to note that the excess Rb II charge gained over $10 \mathrm{GeV}$ in energy (peak energy of about $10.7 \mathrm{GeV}$ was observed in this simulation) and in principle could be observed in the experiment.

\section{CONCLUSIONS}

In summary, an electron beam produced Rb plasma was investigated as a source for plasma wakefield acceleration. This source was explored for mitigating the problems of head erosion and ion motion. However, if the experiment is operated in the blowout regime at a high plasma density such that the drive beam can strongly pinch down and ionize the $\mathrm{Rb}$ further, $\mathrm{Rb}$ II electrons can be injected into the wake and act as dark current. Beam loading by accumulation of the $\mathrm{Rb}$ II electrons can severely reduce the accelerating field and thereby reduce the average transformer ratio in such a plasma. The PIC simulations have confirmed the physical picture emerging from the experimental results. Simulation values for the transformer ratio, and the excess charge are consistent with those seen in the experiment.

\section{ACKNOWLEDGMENTS}

The work at UCLA was supported by NSF Grant No. PHY-1415386 and DOE Grant No. DE-SC0010064. The simulations were carried out on the Hoffman cluster at UCLA. Work at SLAC was supported by Department of Energy Contract No. DE-AC02-76SF00515.

[1] I. Blumenfeld et al., Energy doubling of 42 ?GeV electrons in a metre-scale plasma wakefield accelerator, Nature (London) 445, 741 (2007).

[2] W. Lu, C. Huang, M. Zhou, W. B. Mori, and T. Katsouleas, Nonlinear Theory for Relativistic Plasma Wakefields in the Blowout Regime, Phys. Rev. Lett. 96, 165002 (2006).

[3] J. Rosenzweig, B. Breizman, T. Katsouleas, and J. Su, Acceleration and focusing of electrons in two-dimensional nonlinear plasma wake fields, Phys. Rev. A 44, R6189 (1991).

[4] W. Lu, C. Huang, M. Zhou, M. Tzoufras, F. S. Tsung, W. B. Mori, and T. Katsouleas, A nonlinear theory for multidimensional relativistic plasma wave wakefields, Phys. Plasmas 13, 056709 (2006).

[5] I. Blumenfeld, Scaling of the longitudinal electric fields and transformer ratio in a nonlinear plasma wakefield accelerator, Ph.D. thesis, Stanford University, 2009.

[6] I. Blumenfeld et al., Scaling of the longitudinal electric field and transformer ratio in a nonlinear plasma wakefield accelerator, Phys. Rev. ST Accel. Beams 13, 111301 (2010).

[7] M. M. Zhou, Accelerating ultrashort electron/positron bunches in field ionization produced plasmas, $\mathrm{Ph} . \mathrm{D}$. thesis, University of California, Los Angeles, 2008.

[8] R. Gholizadeh, T. Katsouleas, P. Muggli, C. Huang, and W. Mori, Preservation of Beam Emittance in the Presence of Ion Motion in Future High-Energy Plasma-WakefieldBased Colliders, Phys. Rev. Lett. 104, 155001 (2010).

[9] J. Rosenzweig, A. Cook, A. Scott, M. Thompson, and R. Yoder, Effects of Ion Motion in Intense Beam-Driven Plasma Wakefield Accelerators, Phys. Rev. Lett. 95, 195002 (2005).

[10] B. W. Montague and W. Schnell, Multiple scattering and synchrotron radiation in the plasma beat-wave accelerator, AIP Conf. Proc. 130, 146 (1985). 
[11] C. Clayton et al., Transverse Envelope Dynamics of a 28.5-GeV Electron Beam in a Long Plasma, Phys. Rev. Lett. 88, 154801 (2002).

[12] M. Tzoufras, W. Lu, F. Tsung, C. Huang, W. Mori, T. Katsouleas, J. Vieira, R. Fonseca, and L. Silva, Beam Loading in the Nonlinear Regime of Plasma-Based Acceleration, Phys. Rev. Lett. 101, 145002 (2008).

[13] K. L. F. Bane, P. Chen, and P. B. Wilson, On collinear wake field acceleration, IEEE Trans. Nucl. Sci. 32, 3524 (1985).

[14] R. D. Ruth, A. W. Chao, P. L. Morton, and P. B. Wilson, A Plasma wake field accelerator, Part. Accel. 17, 171 (1985).

[15] B. Jiang, C. Jing, P. Schoessow, J. Power, and W. Gai, Formation of a novel shaped bunch to enhance transformer ratio in collinear wakefield accelerators, Phys. Rev. ST Accel. Beams 15, 011301 (2012).

[16] W. An, W. Lu, C. Joshi, W. B. Mori, C. Huang, M. J. Hogan, S. F. Martins, L. O. Silva, S. H. Gold, and G. S. Nusinovich, Simulations of two-bunch plasma wakefield accelerator experiments at FACET., in Advanced Accelerator Concepts: 14th Advanced Accelerator Concepts Workshop (American Institute of Physics, New York, 2010), pp. 472-477.

[17] M. Litos et al., High-efficiency acceleration of an electron beam in a plasma wakefield accelerator, Nature (London) 515, 92 (2014).

[18] E. Oz et al., Ionization-Induced Electron Trapping in Ultrarelativistic Plasma Wakes, Phys. Rev. Lett. 98, 084801 (2007).

[19] A. Pak, K. A. Marsh, S. F. Martins, W. Lu, W. B. Mori, and C. Joshi, Injection and Trapping of Tunnel-Ionized Electrons into Laser-Produced Wakes, Phys. Rev. Lett. 104, 025003 (2010).

[20] N. Kirby et al., Transverse emittance and current of multi$\mathrm{GeV}$ trapped electrons in a plasma wakefield accelerator, Phys. Rev. ST Accel. Beams 12, 051302 (2009).

[21] M. J. Hogan et al., Plasma wakefield acceleration experiments at FACET, New J. Phys. 12, 055030 (2010).

[22] P. Muggli, K. Marsh, S. Wang, C. Clayton, S. Lee, T. Katsouleas, and C. Joshi, Photo-ionized lithium source for plasma accelerator applications, IEEE Trans. Plasma Sci. 27, 791 (1999).

[23] K. A. Olive et al. (Particle Data Group), Review of particle physics, Chin. Phys. C 38, 090001 (2014).

[24] K. A. Marsh et al., X-ray radiation and electron injection from beam envelope oscillations in plasma wakefield accelerator experiments at FACET., in Proceedings of PAC2013, Pasadena, 2013, (JACoW, Geneva, 2014), pp. 1105-1107.

[25] J. B. Rosenzweig and P. Chen, Beam optics of a selffocusing plasma lens, Phys. Rev. D 39, 2039 (1989).
[26] A. S. Muller, Description of beam-matter interaction in the covariance matrix formalism: Application to modification of emittance and Twiss parameters; revised version, Technical Report No. CERN-PS-2001-013-AE, CERN, Geneva, 2001.

[27] S.Z. Li et al., Head erosion with emittance growth in PWFA, in Advanced Accelerator Concepts: 15th Advanced Accelerator Concepts Workshop (American Institute of Physics, New York, 2012), pp. 582-587.

[28] M. J. Hogan et al., Multi-GeV Energy Gain in a PlasmaWakefield Accelerator, Phys. Rev. Lett. 95, 054802 (2005).

[29] Z. Wu, A. S. Fisher, J. Goodfellow, M. Fuchs, D. Daranciang, M. Hogan, H. Loos, and A. Lindenberg, Intense terahertz pulses from SLAC electron beams using coherent transition radiation?, Rev. Sci. Instrum. 84, 022701 (2013).

[30] S. Wang et al., X-Ray Emission from Betatron Motion in a Plasma Wiggler, Phys. Rev. Lett. 88, 135004 (2002).

[31] D. Johnson et al., Positron Production by X-rays Emitted by Betatron Motion in a Plasma Wiggler, Phys. Rev. Lett. 97, 1 (2006).

[32] R. Fulton, J. Haggerty, R. Jared, R. Jones, J. Kadyk, C. Field, W. Kozanecki, and W. Koska, A high resolution wire scanner for micron-size profile measurements at the SLC, Nucl. Instrum. Methods Phys. Res., Sect. A 274, 37 (1989).

[33] C. O'Connell et al., Plasma production via field ionization, Phys. Rev. ST Accel. Beams 9, 101301 (2006).

[34] D. L. Bruhwiler, D. A. Dimitrov, J. R. Cary, E. Esarey, W. Leemans, and R. E. Giacone, Particle-in-cell simulations of tunneling ionization effects in plasma-based accelerators, Phys. Plasmas 10, 2022 (2003).

[35] K. Marsh et al., Beam matching to a plasma wake field accelerator using a ramped density profile at the plasma boundary, in Proceedings of the 21st Particle Accelerator Conference, Knoxville, 2005 (IEEE, Piscataway, NJ, 2005), pp. 2702-2704.

[36] I. Kostyukov, S. Kiselev, and A. Pukhov, X-ray generation in an ion channel, Phys. Plasmas 10, 4818 (2003).

[37] N. Vafaei-Najafabadi, Experimental investigations of beam-driven plasma wakefield accelerators, Ph.D. thesis, University of California, Los Angeles, 2016.

[38] N. Vafaei-Najafabadi et al., Beam Loading by Distributed Injection of Electrons in a Plasma Wakefield Accelerator, Phys. Rev. Lett. 112, 025001 (2014).

[39] R. A. Fonseca, L. O. Silva, F. S. Tsung, V. K. Decyk, W. Lu, C. Ren, W. B. Mori, S. Deng, S. Lee, T. Katsouleas, and J.C. Adam, OSIRIS: A three-dimensional, fully relativistic particle in cell code for modeling plasma based accelerators, Lect. Notes Comput. Sci. 2331, 342 (2002). 